\title{
Common Fixed Point for R-Weak Commutative Mappings without Continuity in Fuzzy Metric Spaces
}

\author{
Deepti Thakur and Rajinder Sharma \\ Department of General Requirements \\ P.O. Box: 135, Code: 311, College of Applied Sciences - Sohar, Oman
}

\begin{abstract}
This paper deals with some results on common fixed point theorems in fuzzy metric spaces generalizing the earlier results of Pant [20], Som [28], [29] and Vasuki [30] by removing the assumption of continuity.
\end{abstract}

\section{General Terms}

$47 \mathrm{H} 10,54 \mathrm{H} 25$

\section{Keywords}

Common fixed point, Fuzzy metric space, R-weak commutative mappings.

\section{INTRODUCTION}

Zadeh [31] paves the way for fuzzy mathematics by introducing the concept of fuzzy sets. Deng [7], Erceg [8], Kaleva and Seikkala [16], Kramosil and Michalek [17] have introduced the concepts of fuzzy metric spaces in different ways. Grabiec [10] followed Kramosil and Michalek [17] and obtained the fuzzy version of Banach's fixed point theorem. The most interesting references in this direction are : George and Veeramani [9], Kaleva [15], Mishra, Sharma and Singh [19], Sharma [22],[23], Sharma and Bagwan [24], Sharma and Deshpande [25],[26],[27], Cho [5] and for fuzzy mappings : Bose and Sahani [1], Lee, Cho and Jung [18], Butnariu [2], Heilpern [11], Chang [3], Chang, Cho, Lee and Lee [4]. In 1976, Jungck [12] established common fixed point theorems for commuting maps generalizing the Banach's fixed point theorem. Sessa [21] defined a generalization of commutavity, which is called weak commutativity. Further, Jungck [13] introduced more generalized commutativity, so called compatibility. Mishra, Sharma and Singh [19] introduced the concept of compatibility in fuzzy metric spaces. In 1998, Jungck and Rhoades [14] introduced the notion of weakly compatible maps and showed that compatible maps are weakly compatible but converse need not true. Sharma and Deshpande [26] improved the results of Mishra, Sharma and Singh [19], Cho [5], Cho Pathak, Kang \& Jung [6], Sharma [22] and Sharma \& Deshpande [25]. They proved common fixed point theorems for weakly compatible maps in fuzzy metric spaces without taking any mapping continuous.

\section{PRELIMINARIES}

Definition 2.1 [31] A fuzzy set $\mathrm{A}$ in $\mathrm{X}$ is a function with domain $\mathrm{X}$ and values in $[0,1]$.

Definition 2.2 [27] A binary operation $*:[0,1] \times[0,1] \rightarrow$ $[0,1]$ is a continuous t-norm if $\{[0,1], *\}$ is an abelian topological monoid with unit 1 such that $a * b \leq c * d$

whenever $a \leq c$ and $b \leq d, a, b, c, d \in[0,1]$.

Definition 2.3 [17] The triplet $(X, M, *)$ is a fuzzy metric space if $X$ is an arbitrary set, $*$ is continuous $t$-norm. $M$ is a fuzzy set in $X^{2} \times[0, \infty]$ satisfying the following conditions:

$$
\begin{array}{ll}
(F M-1) & M(x, y, 0)=0 . \\
(F M-2) & M(x, y, t)=1, \text { for all } t>0 \Leftrightarrow x=y \\
(F M-3) & M(x, y, t)=M(y, x, t) . \\
(F M-4) & M(x, y, t) * M(y, z, s) \leq M(x, z, t+s) \\
& \text { for all } x, y, z \in X \text { and } t, s>0 . \\
(F M-5) & M(x, y, .):[0, \infty] \rightarrow[0,1] \text { is left continuous. }
\end{array}
$$

In this paper $(X, M, *)$ will denote a fuzzy metric space in the sense of above definition with the following condition

$(F M-6) \lim _{n \rightarrow \infty} M(x, y, t)=1$ for all $x, y \in X$.

Definition 2.4 [17] A sequence $\left\{x_{n}\right\}$ in a fuzzy metric space $(X, M, *)$ is called Cauchy sequence if

$$
\lim _{n \rightarrow \infty} M\left(x_{n+p}, x_{n}, t\right)=1
$$

for every $t>0$ and each $p>0$.

Definition 2.5 [17] A sequence $\left\{x_{n}\right\}$ in a fuzzy metric space

(X, $\mathrm{M}, *$ ) is said to be convergent to $x \in X$ if

$$
\lim _{n \rightarrow \infty} M\left(x_{n} x, t\right)=1 \text { for each } t>0 .
$$

Definition 2.6 [17] A fuzzy metric space $(X, M, *)$ is said to be complete if every Cauchy sequence in $X$ converges in $X$.

Remark 2.1 Since $*$ is continuous, it follows from $(F M-4)$ that limit of sequence is uniquely determined.

Lemma 2.1 [5] Let $\left\{y_{n}\right\}$ be a sequence in a fuzzy metric space $(X, M, *)$ with the condition $(F M-6)$. If there exists a number $k \in(0,1)$ such that

$$
\begin{aligned}
& \qquad M\left(y_{n+2}, y_{n+1}, k t\right) \geq M\left(y_{n+1}, y_{n}, t\right) \\
& \text { for all } t>0 \text { and } n=1,2, \ldots \\
& \text { then }\left\{y_{n}\right\} \text { is a Cauchy sequence in } X \text {. }
\end{aligned}
$$

Lemma 2.2 [19] If for all $x, y \in X, t>0$ and for a number $k \in(0,1)$

$$
M(x, y, k t) \geq M(x, y, t) \text { then } x=y .
$$

Definition 2.7 [19] Let A and B be mappings from a fuzzy metric space $(X, M, *)$ into itself. The mappings $\mathrm{A}$ and $\mathrm{B}$ are said to be compatible if

$$
\lim _{n \rightarrow \infty} M\left(A B x_{n}, B A x_{n}, t\right)=1, \text { for all } t>0,
$$

whenever $\left\{x_{n}\right\}$ is a sequence in $\mathrm{X}$ such that

$$
\lim _{n \rightarrow \infty} A x_{n}=\lim _{n \rightarrow \infty} B x_{n}=z \text { for some } z \in X .
$$

Definition 2.8[14] A pair $A$ and $S$ is called weakly compatible pair in fuzzy metric space if they commute at a coincidence points. 
Example 2.1 Let $X=[0,2]$ with the metric $d$ defined by $d(x, y)=|x-y|$

for each $t \in(0, \infty)$, define

$$
M(x, y, t)=\frac{t}{t+d(x, y)}, \quad x, y \in X
$$

and define

$$
M(x, y, 0)=0, \quad x, y \in X .
$$

Clearly $(X, M, *)$ is a fuzzy metric space on $X$ where $*$ is defined by

$$
a * b=a b \text { or } a * b=\min \{a, b\} .
$$

Define $A, B: X \rightarrow X$ by

$$
A x=\left\{\begin{array}{c}
x, \text { if } x \in\left[0, \frac{1}{4}\right) \\
\frac{1}{4}, \text { if } x \geq \frac{1}{4}
\end{array} \text { and } B x=\frac{x}{1+x},\right.
$$

for all $x \in[0,2]$.Consider the sequence $\left\{x_{n}=\frac{1}{3}+\frac{1}{n}: n \geq 1\right\}$ in $X$. Then

$$
\lim _{n \rightarrow \infty} A x_{n}=\frac{1}{4}, \lim _{n \rightarrow \infty} B x_{n}=\frac{1}{4}
$$

But

$$
\lim _{n \rightarrow \infty} M\left(A B x_{n}, B A x_{n}, t\right)=\frac{t}{t+\left|\frac{1}{4}-\frac{1}{5}\right|} \neq 1 .
$$

Thus $A$ and $B$ are non-compatible. But $A$ and $B$ are commuting at their coincidence point $x=0$, that is weakly compatible at $x=0$.

Thus, weakly compatible maps need not be compatible.

Definition 2.9 [20] Two mappings $f$ and $g$ of a fuzzy metric space $(X, M, *)$ into itself are said to be weakly commuting if

$$
M(f g x, g f x, t) \geq M(f x, g x, t), \text { for every } x \in X .
$$

Definition 2.10 [30] The mappings $f$ and $g$ of a fuzzy metric space $(\mathrm{X}, \mathrm{M}, *)$ into itself are $\mathrm{R}$-weakly commuting provided there exists some positive real number $\mathrm{R}$ such that

$$
M(f x, g f x, t) \geq M\left(f x, g x, \frac{t}{R}\right), \text { for all } x \in X .
$$

Weak commutativity implies R-weak commutativity and the converse is true for $R \leq 1$.

\section{MAIN RESULTS}

Theorem 3.1 Let $S$ and $T$ be two self mappings of a fuzzy metric space $(X, M, *)$. Let $A$ be a self mapping of $X$ satisfying

(3.1) $A(X) \subseteq S(X)$ and $A(X) \subseteq T(X)$

(3.2) $M(A x, A y, t) \geq r\left[\min \left\{\begin{array}{l}M(S x, T y, t), M(S x, A x, t), \\ M(S x, A y, t), M(T y, A y, t)\end{array}\right]\right.$.

for all $x, y \in X$ where $r:[0,1] \rightarrow[0,1]$ is a continuous function such that

$$
r(t)>t \text { for each } t<1 \text { and } r(t)=1 \text { for } t=1 .
$$

(3.3) If one of $A(X), S(X), T(X)$ is a complete subspace of $X$,

then

(i) $\quad A$ and $S$ have a coincidence point, and

(ii) $\quad A$ and $T$ have a coincidence point.
Further if

(3.4) $\{A, S\}$ and $\{A, T\}$ are $R$-weakly commuting mappings, then

(iii) $\quad A, S$ and $T$ have a unique common fixed point in $X$.

Proof. Let a sequence $\left\{y_{n}\right\}$ in $X$ be such that

$$
\begin{aligned}
& y_{2 n}=A x_{2 n}=S x_{2 n+1} \\
& y_{2 n+1}=A x_{2 n+1}=T x_{2 n+2}
\end{aligned}
$$

and $T(X)$ be complete. Note that the subsequence $\left\{y_{2 n+1}\right\}$ is contained in $T(X)$ and has a limit in $T(X)$, call it $z$.

Let $w \in T^{-1}(z)$, then $T w=z$.

We shall use the fact that subsequences $\left\{y_{2 n}\right\},\left\{y_{2 n+2}\right\}$ also converges to $z$.

By putting $x=x_{2 n+1}, y=w$ in (3.2), we get

$$
\left.\begin{array}{rl}
M\left(A x_{2 n+1}, A w, t\right) & =M\left(y_{2 n+1}, A w, t\right) \\
\geq r\left[\min \left\{\begin{array}{c}
M\left(y_{2 n}, T w, t\right), M\left(y_{2 n}, y_{2 n+1}, t\right), \\
M\left(y_{2 n}, A w, t\right), M(T w, A w, t)
\end{array}\right]\right.
\end{array}\right]
$$

Taking limit as $n \rightarrow \infty$, we get

$$
M(z, A w, t) \geq r M(z, A w, t)>M(z, A w, t)
$$

which is a contradiction. Therefore $A w=T w=z$ i.e. $w$ is a coincidence point of $A$ and $T$.

Since $A(X) \subset S(X), T w=z$ implies that $z \in S(X)$.

Let $v \in S^{-1} z$. Then $S v=z$.

By putting $x=v$ and $y=x_{2 n+2}$ in (3.2), we get

$$
\begin{aligned}
& M\left(A v, A x_{2 n+2}, t\right)=M\left(A v, y_{2 n+2}, t\right) \\
& \geq r\left[\min \left\{\begin{array}{c}
M\left(S v, y_{2 n+1}, t\right), M(S v, A v, t), \\
M\left(S v, y_{2 n+2}, t\right), M\left(y_{2 n+1}, y_{2 n+2}, t\right)
\end{array}\right\}\right]
\end{aligned}
$$

Taking limit as $n \rightarrow \infty$, we get

$$
M(A v, z, t) \geq r M(z, A v, t)>M(z, A v, t)
$$

which is a contradiction. Therefore $A v=S v=z$ i.e. $v$ is a coincidence point of $A$ and $S$.

If $A(X)$ is complete then by (3.1) $z \in A(X) \subset T(X)$ or

$z \in A(X) \subset S(X)$.

Thus (i) and (ii) are completely established.

Since the pair $\{A, T\}$ is R-weakly commuting, therefore we have

$$
M(A T w, T A w, t) \geq M\left(A w, T w, \frac{t}{R}\right), \text { for all } x \in X
$$

which gives $A T w=T A w$, i.e. $A z=T z$.

Similarly the R-weak commutativity of pair $\{A, S\}$ gives $A z=S z$.

By putting $x=x_{2 n+1}, y=z$ in 3.2 we get

$M\left(A x_{2 n+1}, A z, t\right)=M\left(y_{2 n+1}, A z, t\right)$

$$
\geq r\left[\min \left\{\begin{array}{c}
M\left(S x_{2 n+1}, T z, t\right), M\left(S x_{2 n+1}, A x_{2 n+1}, t\right), \\
M\left(S x_{2 n+1}, A z, t\right), M(T z, A z, t)
\end{array}\right\}\right] .
$$

Taking limit as $n \rightarrow \infty$, we get

$$
M(z, A z, t) \geq r M(z, A z, t)>M(z, A z, t),
$$


which is a contradiction. Thus $A z=z=S z=T z$ i.e. $z$ is common fixed point of $A, S$ and $T$.

Theorem 3.2 Let $S$ and $T$ be two self mappings of a fuzzy metric space $(X, M, *)$. Let $A, B, S$ and $T$ be self mappings of $X$ satisfying

(3.5) $A(X) \subseteq S(X)$ and $B(X) \subseteq T(X)$.

(3.6) $\quad a M(T x, S y, t)+b M(T x, A x, t)+c M(S y, B y, t)+$ $\max \{M(A x, S y, t), M(B y, T x, t)\} \leq q M(A x, B y, t)$, for all $x, y \in X$ where $a, b, c \geq 0, q>0$ with $q<a+b+c+1$.

(3.7) If one $A(X), B(X), S(X), T(X)$ is complete subspace of $X$ then

(i) $\quad A$ and $T$ have a coincidence point, and

(ii) $B$ and $S$ have a coincidence point,

Further if

(3.8) $\{A, T\}$ and $\{B, S\}$ are $R$-weakly commuting pairs, then

(iii) $A, B, S$ and $T$ have a unique common fixed point in $X$.

Proof. Suppose that $T(X)$ is complete. Note that the subsequence $\left\{y_{2 n+1}\right\}$ is contained in $T(X)$ and has a limit in $T(X)$, call it $z$.

Let $w \in T^{-1}(z)$, then $T w=z$.

We shall use the fact that subsequence $\left\{y_{2 n}\right\}$ also converges to

By putting $x=w$ and $y=x_{2 n+1}$ in (3.6), we get

$a M\left(T w, y_{2 n}, t\right)+b M(T w, A w, t)+c M\left(y_{2 n}, y_{2 n+1}, t\right)$

$+\max \left\{\begin{array}{c}M\left(A w, y_{2 n}, t\right), \\ M\left(y_{2 n+1}, T w, t\right)\end{array}\right\} \leq q M\left(A w, y_{2 n+1}, t\right)$.

As $n \rightarrow \infty$, we get

$$
M(A w, z, t) \geq \frac{a+c}{q-b-1}>1,
$$

which is a contradiction.

Thus, $A w=z=T w$, i.e. $w$ is a coincidence point of $A$ and $T$.

Since $A(X) \subseteq S(X), A w=z$ implies that $z \in S(X)$.

Let $v \in S^{-1} z$. Then $S v=z$.

By putting $x=x_{2 n+2}, y=v$ in (3.6), we get

$$
\begin{aligned}
& a M\left(y_{2 n+1}, S v, t\right)+b M\left(y_{2 n+1}, y_{2 n+2}, t\right)+c M(S v, B v, t) \\
& +\max \left\{\begin{array}{l}
M\left(y_{2 n+2}, S v, t\right), \\
M\left(B v, y_{2 n+1}, t\right)
\end{array}\right\} \leq q M\left(y_{2 n+2}, B v, t\right) .
\end{aligned}
$$

As $n \rightarrow \infty$, we have

$$
M(B v, z, t) \geq \frac{a+b}{q-c-1}>1,
$$

a contradiction. Therefore $B v=z=S v$, i.e. $v$ is a coincidence point of $B$ and $S$.

If $A(X)$ or $B(X)$ is complete then by (3.5)

$$
z \in A(X) \subseteq S(X) \text { or } z \in B(X) \subseteq T(X) .
$$

Thus (i) and (ii) are completely established.

Since the pair $\{A, T\}$ is $R$-weakly commuting therefore we have

$$
M(A T w, T A w, t) \geq M(A w, T w, t) .
$$

which gives $A T w=T A v$ i.e. $A z=T z$.

Similarly $B z=S z$.

By putting $x=z, y=x_{2 n+1}$ in (3.6)

$$
\begin{aligned}
& a M\left(T z, y_{2 n}, t\right)+b M(T z, A z, t)+c M\left(y_{2 n}, y_{2 n+1}, t\right)+ \\
& \max \left\{\begin{array}{c}
M\left(A z, y_{2 n}, t\right), \\
M\left(y_{2 n+1}, T z, t\right)
\end{array}\right\} \leq q M\left(A z, y_{2 n+1}, t\right),
\end{aligned}
$$

As $n \rightarrow \infty$, we have

$$
M(A z, z, t) \geq \frac{b-c}{q-a-1}<1,
$$

which is a contradiction. Thus $A z=z=B z=S z=T z$.

\section{CONCLUSION}

The theorems in this paper are the improved ,extended and generalized form of some earlier results on common fixed point theorems in fuzzy metric spaces given by Pant[20], vasuki [30], and som [28],[29]. The proven results in fuzzy metric spaces for $\mathrm{R}$-weak commutative mappings without taking any mapping continuous shows that for existence of fixed point in fuzzy metric space , continuity of any mapping is not needed.

\section{ACKNOWLDGMNTS}

The authors are thankful to Prof. Sushil Sharma for his valuable suggestions during the preparation of this paper.

\section{REFERENCES}

[1] Bose, B. K. and Sahani, D. Fuzzy mappings and fixed point theorems. Fuzzy Sets and Systems, 21 (1987), 5358.

[2] Butnariu, D. Fixed points for fuzzy mappings. Fuzzy Sets and Systems, 7(1982), 191-207.

[3] Chang, S. S. Fixed point theorem for fuzzy mappings. Fuzzy Sets and Systems, 17(1985), 181-187.

[4] Chang, S. S., Cho, Y. J., Lee, B.S. and Lee, G. M. Fixed degree and fixed point theorems for fuzzy mappings. Fuzzy Sets and Systems, 87(3) (1997), 325-334.

[5] Cho, Y. J. Fixed points in fuzzy metric spaces. J. Fuzzy Math. 5(4) (1997), 949- 962.

[6] Cho, Y. J., Pathak, H.K., Kang, S. M. and Jung, J. S. Common fixed points of compatible maps of type $(\beta)$ on fuzzy metric spaces. Fuzzy Sets and Systems, 93(1998), 99-111.

[7] Deng, Z. K. Fuzzy pseudo metric spaces. J. Math. Anal Appl. 86(1982), 74-95.

[8] Ercez, M.A. A metric space in fuzzy set theory. J. Math. Anal. Appl. 69 (1979), 205-230.

[9] George, A. and Veeramani, P. On some results in fuzzy metric spaces. Fuzzy Sets and Systems, 64 (1994), 395399.

[10] Grabiec, M. Fixed point in fuzzy metric space. Fuzzy Sets and Systems, 27(1988), 385-389. 
[11] Heilpern, S. Fuzzy mappings and fixed point theorems. J. Math. Anal. Appl. 83(1981), 566-569.

[12] Jungck, G. Commuting mappings and fixed points. Amer. Math. Monthly, 83(1976), 261-263.

[13] Jungck, G. Compatible mappings and common fixed points. Internat. J. Math. Math. Sci. 9(1986), 771-779.

[14] Jungck, G., and Rhoades, B. E. Fixed point for set valued functions without continuity. Indian Journal of Pure and Applied Maths. 29(3)(1998), 227-238.

[15] Kaleva, O. The completion of fuzzy metric spaces. J.Math. Anal. Appl. 109(1985), 194- 198.

[16] Kaleva, O. and Seikkala, S. On fuzzy metric spaces. Fuzzy Sets and Systems, 12(1984), 215-229.

[17] Kramosil, I. and Michalek, J. Fuzzy metric and statistical metric spaces. Kybernetika, 11(1975), 336344.

[18] Lee, B. S., Cho, Y. J. and Jung, J. S. Fixed point theorems for fuzzy mappings and application. Comm. Korean Math. Sci. 11(1966), 89-108.

[19] Mishra, S. N., Sharma, N. and Singh, S. L. Common fixed points of maps in fuzzy metric spaces. Internat. J. Math. Math. Sci. 17(1994), 253-258.

[20] Pant, R. P. R-weak commutativity and fixed points. Soochoo J. Math. 25(1999), 37-42.

[21] Sessa, S. On weak commutativity condition of mappings in a fixed points considerations. Publ. Inst. Mat. 32(46) (1982), 149-153.
[22] Sharma, Sushil Common fixed point theorems in fuzzy metric spaces. Fuzzy set. syst. 125 (2001), 1-8.

[23] Sharma, Sushil Common fixed point theorems in fuzzy metric space. Fuzzy Sets and Systems, 127 (2002), 345352.

[24] Sharma, Sushil and Bagwan, A. Common fixed point theorem for six mappings in Menger spaces. Fasciculi Mathematici, 37(2007), 67-77.

[25] Sharma, Sushil and Deshpande, B. Common fixed point theorems for contractive and R- weakly commuting maps. J. Bangladesh Acad. Sci. 25, 2 (2001), $1-9$.

[26] Sharma, Sushil and Deshpande, B. Common fixed point for weakly compatible mappings without continuity in fuzzy metric spaces. East Asian Math. J. 18 2 (2002) 183-193.

[27] Sharma, Sushil and Deshpande, B. Common fixed points without continuity in fuzzy metric spaces. J. Pure \& Applied Math. 12, 4 (2005), 289-306.

[28] Som, T. Some fixed point theorems on metric and Banach spaces. Indian J. Pure Appl. Math. 16:6 (1985), 575-585.

[29] Som, T. Some results on common fixed point in fuzzy metric spaces. Soochow J. Math. 33:4 (2007), 553-561.

[30] Vasuki, R. Common fixed points for R-weakly commuting maps in fuzzy metric spaces. Indian J. Pure Appl. Math.30: 4 (1999), 419-423.

[31] Zadeh, L.A. Fuzzy sets Inform Control 8(1965), 338353. 